\title{
Nanofabrication of Tailored Surface Structures in Dielectrics Using Temporally Shaped Femtosecond-Laser Pulses
}

\author{
Javier Hernandez-Rueda, ${ }^{* \dagger}$ Nadine Götte, ${ }^{\S}$ Jan Siegel, ${ }^{* \dagger}{ }^{\dagger}$ Michelina Soccio, ${ }^{\ddagger}$ Bastian Zielinski, ${ }^{\S}$ \\ Cristian Sarpe, ${ }^{\S}$ Matthias Wollenhaupt, ${ }^{\S, \|}$ Tiberio A. Ezquerra, ${ }^{\ddagger}$ Thomas Baumert, ${ }^{\S}$ and Javier Solis ${ }^{\dagger}$ \\ ${ }^{\dagger}$ Instituto de Óptica and ${ }^{\ddagger}$ Instituto de Estructura de la Materia, Consejo Superior de Investigaciones Científicas, Serrano 121,28006 \\ Madrid, Spain \\ ${ }^{\S}$ Institut für Physik, Universität Kassel, Heinrich-Plett-Strasse 40, 34132 Kassel, Germany \\ "Carl von Ossietzky Universität Oldenburg, Institut für Physik, Carl-von-Ossietzky-Straße 9-11, 26129 Oldenburg, Germany
}

ABSTRACT: We have investigated the use of tightly focused, temporally shaped femtosecond (fs)-laser pulses for producing nanostructures in two dielectric materials (sapphire and phosphate glass) with different characteristics in their response to pulsed laser radiation. For this purpose, laser pulses shaped by third-order dispersion (TOD) were used to generate temporally asymmetric excitation pulses, leading to the singlestep production of subwavelength ablative and subablative surface structures. When compared to previous works on the interaction of tightly focused TOD-shaped pulses with fused silica, we show here that this approach leads to very different nanostructure morphologies, namely, clean nanopits without debris surrounding the crater in sapphire and well-outlined nanobumps and nanovolcanoes in phosphate glass. Although in sapphire the debris-free processing is associated with the much lower viscosity of the melt compared to fused silica, nanobump formation in phosphate glass is caused by material network expansion (swelling) upon resolidification below the ablation threshold. The formation of nanovolcanoes is a consequence of the combined effect of material network expansion and ablation occurring in the periphery and central part of the irradiated region, respectively. It is shown that the induced morphologies can be efficiently controlled by modulating the TOD coefficient of the temporally shaped pulses.

KEYWORDS: temporal pulse shaping, femtosecond lasers, dielectrics, phosphate glass, sapphire, nanofabrication

\section{INTRODUCTION}

Within the last two decades, ultrashort laser pulses have been widely used for materials processing, covering a broad range of technological applications. ${ }^{1-5}$ This is particularly relevant for the case of dielectrics, for which the high peak power and short pulse duration associated with ultrafast lasers trigger multiphoton (MPI) and avalanche ionization (AI) processes for efficiently coupling the laser energy into the material. The highly deterministic nature of MPI allows energy coupling into the material with a spatial resolution without precedents (few tens of nanometers). ${ }^{6}$ AI builds on the presence of seed electrons produced by MPI, which are accelerated by the light field and multiplied via impact ionization, leading to a strongly enhanced absorption. The relative balance of these ionization mechanisms is given foremost by the laser pulse duration/shape that strongly influences the final crater morphology. ${ }^{7}$ The resulting complex excitation dynamics have been investigated by monitoring the evolution of the optical properties of the material with femtosecond resolution. ${ }^{8-12}$ In particular, temporal pulse shaping has emerged as a powerful tool for investigating and controlling the underlying carrier excitation
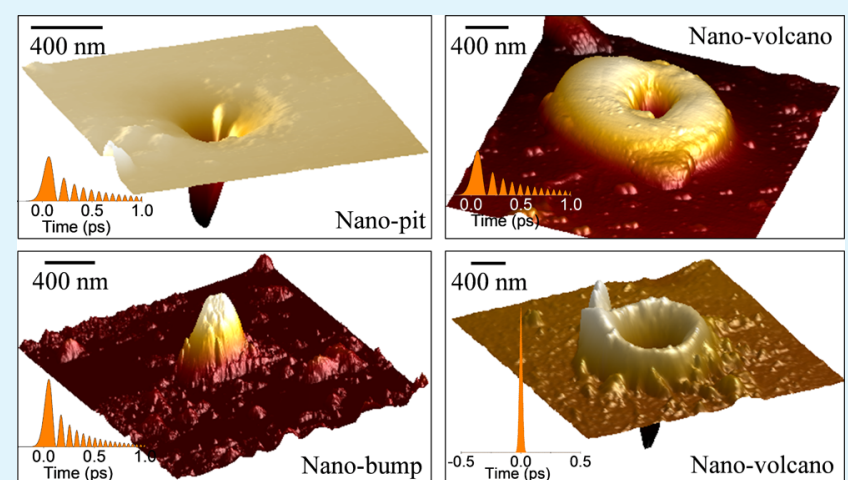

and relaxation mechanisms, in some cases yielding results that cannot be achieved by using transform-limited (TL) pulses. ${ }^{6,13-19}$ It has been shown that certain symmetric and asymmetric pulse shapes provide superior structuring results in dielectrics. ${ }^{7,13-18}$ In this respect, the results associated with the use of tightly focused, temporally nonsymmetric pulse trains for surface structuring of fused silica are particularly significant. $^{7,14-16}$ The craters produced with third-order dispersion TOD-shaped pulses with a decreasing intensity envelope showed a diameter reduction of one order of magnitude $\left(D_{\mathrm{TOD}} \approx 100 \mathrm{~nm}\right)$ compared with the values achieved for TL pulses with the same integrated energy $\left(D_{\mathrm{TL}} \approx 1 \mu \mathrm{m}\right) .{ }^{14,15}$ The inspection of the surface topography by atomic force microscopy (AFM) revealed that these narrow craters are surrounded by a protruded rim likely caused by the flow of molten material out of the crater caused by the recoil pressure exerted by the ablating material. ${ }^{16,20}$ Besides, these studies

Received: December 18, 2014

Accepted: March 11, 2015 
reported a different ablation threshold for the time-reversed counterpart of the nonsymmetric pulse train, which was explained by the differences in the energy coupling efficiency for both pulse envelopes.

In this work, we have used tightly focused temporally shaped fs-laser pulses to control surface-structuring processes in sapphire and phosphate glass, tailoring the shape and lateral dimension of the so-produced nanostructures. By exploiting the different response of these materials upon irradiation with fslaser pulses (as studied under loose focusing conditions in previous works), ${ }^{9,21-23}$ we have been able to produce structures that are very different from those generated in fused silica (nanopits, nanobumps, and nanovolcanoes with a well-outlined rim). The topography and morphological features of the laserproduced structures have been characterized by AFM and are discussed in terms of the balance between the different ionization mechanisms (MPI and AI), how they are conditioned by the temporal shape, and by the specific material response to fs-laser irradiation. The characteristics of the produced nanostructures are also compared to those produced by other nanostructuring techniques, such as 3D-micromachining, ${ }^{4,24,25}$ 2D-ordered surface array fabrication, $5,26,27$ and surface drilling, ${ }^{28,29}$ in different materials.

\section{EXPERIMENTAL SECTION}

The experimental layout combines a temporal pulse shaper with an automated irradiation setup. ${ }^{30,31}$ The laser source used in this work was a $\mathrm{Ti} /$ sapphire amplifier that delivers $\mathrm{TL}$ pulses of $30 \mathrm{fs}$ pulse duration, at $790 \mathrm{~nm}$ and $0.8 \mathrm{~mJ}$ per pulse. The linearly polarized laser pulse passes through the temporal pulse shaper system, which allows modulation of the laser spectrum (spectral width $\Delta \lambda=30 \mathrm{~nm}$ $(\mathrm{fwhm})$ ) and consequently the temporal shape of the laser pulse. A detailed description of the system is given in refs 14,15 , and 30 . In brief, it combines a $4 \mathrm{f}$ zero-dispersion system and a dual liquid-crystal spatial light modulator (LC-SLM, with two layers of 640 pixels) located at its Fourier plane. The LC-SLM modulates the relative phase of each spectral component of the pulse by changing the birefringence of the liquid crystals at each pixel via electro-optical control. In this way chromatic dispersion can be introduced, which changes the temporal pulse shape. In particular, the spectral phase can be described via the Taylor expansion, with second-, third-, and higher-order terms. The SLM is connected to a personal computer that runs an ad-hocdeveloped software, which allows for individually setting the coefficients of the Taylor expansion of the spectral phase. In this work, only the third-order dispersion coefficient $\varphi^{(3)}$ was used to control the pulse shape because this order allows for design of asymmetric pulse trains with $\varphi^{(3)}$ values ranging from 0 up to $1000 \times$ $10^{3} \mathrm{fs}^{3}$. Afterward, the laser pulse is injected in the irradiation setup, which uses a microscope objective (Zeiss LD Epiplan 50×, NA $=0.5$ ) to focus the beam at the sample surface to a nominal diffractionlimited $1 / \mathrm{e}^{2}$ spot diameter of $1.4 \mu \mathrm{m}\left(D_{\text {nominal }}=2(0.44 \lambda /(\mathrm{NA}))\right) .{ }^{32}$ The pulse shaper system is calibrated by maximizing the signal of a two-photon absorption photodiode (in the plane where the sample will be placed), in order to precompensate the residual spectral phase of the laser pulse, induced by passing through several optical elements (especially the microscope objective). A three-axis motorized translation stage is used for positioning the sample, whereas the laser pulse energy is adjusted by a motor-driven gradient-neutral density filter and measured with a cross-calibrated photodiode. The single-shot, laserexposed regions are distributed in a $2 \mathrm{D}$ array where the energy and the $\mathrm{Z}$ position of the sample are varied in discrete steps $(\Delta E \approx 5-10 \mathrm{~nJ}$, $\Delta z \approx 1-2 \mu \mathrm{m}$ ) along the horizontal and vertical directions of the matrix, respectively. The optimal $\mathrm{Z}$ position, in which the spot size of the laser beam at the surface is minimum, is identified afterward by selecting the line with the lowest (energy) modification threshold for a given pulse form. All irradiations presented here were produced using single laser pulses, therefore no incubation effects caused by multipulse exposure were observed.

For the temporal characterization of the laser pulses, a crosscorrelation technique was used. ${ }^{14,15}$ The spectral phase induced by the SLM was adjusted to maximize the two-photon absorption signal of a photodiode positioned at the sample plane in order to ensure the shortest pulse duration and determine the reference phase for the pulse shaper. For experiments with TOD pulses, the corresponding phase masks with positive coefficients have been applied, which lead to the generation of pulse trains with decreasing intensity envelopes. ${ }^{16}$

We have irradiated two different dielectric materials for which the ablation behavior upon irradiation with 100 fs laser pulses has been already described in detail in literature. ${ }^{9,21-23}$ Namely, sapphire $\left(E_{\mathrm{g}}=\right.$ $9.9 \mathrm{eV}$, from VM-TIM) and an $(\mathrm{Er}, \mathrm{Yb})$ co-doped phosphate glass ( $E_{\mathrm{g}}$ $=3.6 \mathrm{eV}, 2 \% \mathrm{Er}^{3+}, 2.5 \% \mathrm{Yb}^{3+}$ wt $\%$, MM2 from Kigre). Before and after irradiation the samples were cleaned in an ultrasonic bath $(16 \mathrm{~min})$, using different organic and/or inorganic solutions depending on the sample composition (isopropanol, $\mathrm{KOH}$, and $\mathrm{HCl}$ ). Then, the topography of the irradiated regions was characterized by using AFM. A Multimode 8 AFM with a Nanoscope V controller (Bruker) was used in tapping mode with NCHV probes (Bruker). Square images with a $512 \times 512$ pixels resolution were acquired. Analysis of the size and shape of nanostructures was performed with the NanoScope Analysis 1.40 software (Bruker), using a Bruker NCHV tip with front angle $(\mathrm{FA})=25^{\circ}$, back angle $(\mathrm{BA})=15^{\circ}$, and side angle $(\mathrm{SA})=22.5^{\circ}$. A conservative estimation of how deep this tip can probe holes with a narrow diameter can be made using the largest angle (FA $\left.=25^{\circ}\right)$ and the geometric relation, $\tan (\mathrm{FA})=$ crater radius $/$ crater depth $=0.47$.

\section{RESULTS AND DISCUSSION}

3.1. Sapphire. Figure 1a shows the calculated temporal intensity profiles of the laser pulses used to irradiate the sapphire sample, a TL pulse of $30 \mathrm{fs}$ pulse duration (left) and a pulse train (right) generated using a TOD coefficient of $\varphi^{(3)}=$ $600 \times 10^{3} \mathrm{fs}^{3}$. Figure $1 \mathrm{~b}$ displays the corresponding AFM micrographs and topography profiles of the craters. The individual energies used in each case were selected so that similar crater depths were produced. The images illustrate the large decrease in crater diameter when using TOD-shaped pulses. This can be more clearly seen in the crater depth profiles, featuring fwhm-diameters of $D_{\text {fwhm }}(\mathrm{TL})=720$ and $D_{\text {fwhm }}($ TOD $)=164 \mathrm{~nm}$, respectively. In both cases, unlike the case of fused silica, ${ }^{14}$ the crater edge shows a clean perimeter without traces of splashing or material redeposition. The absence of such an elevated rim is most likely related to the low viscosity of molten sapphire $(\eta \approx 0.027 \mathrm{~Pa} \cdot \mathrm{s}$ at $T=2500 \mathrm{~K})$, which is five orders of magnitude smaller than that of fused silica at the same temperature. ${ }^{9,33}$ It has been shown that such low viscosity leads to the formation of a transient elevation at the edge of the crater, caused by the ablating plasma recoil pressure acting on the molten phase. This effect has been studied in fs-microscopy experiments and is evident after a few hundreds of picoseconds. ${ }^{9}$ The much higher viscosity of silica, although impeding a substantial material flow for larger laser spot sizes, ${ }^{9}$ leads in this case to significant viscous flow/ splashing effects when using tight focusing. Our results thus demonstrate that the material choice is crucial when pursuing nanostructuring applications requiring the production of cleanly ablated holes.

Although the crater profile produced by the TL pulse shows a maximum depth of $h=-350 \mathrm{~nm}$, the one produced by the pulse train features two different ablation regimes: a deep and narrow central crater $(h=-450 \mathrm{~nm})$, surrounded by a wider, shallow annular depression $(h=-50 \mathrm{~nm})$. By using pulse trains, it is possible to reduce the crater diameter while 

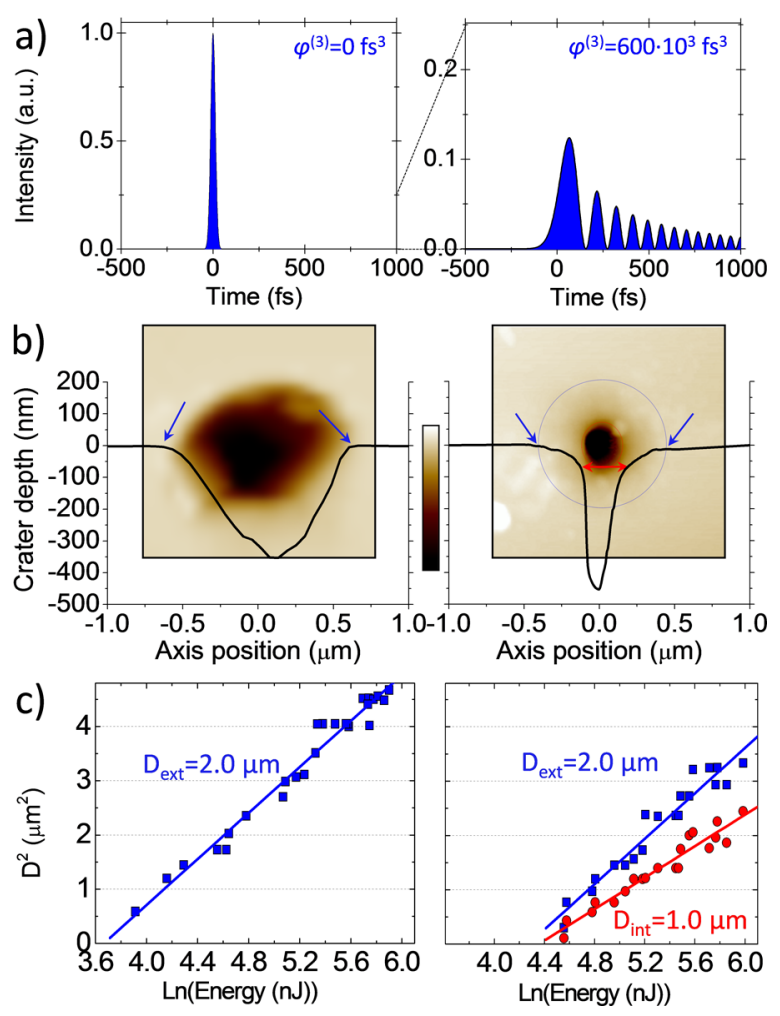

Figure 1. (a) Calculated temporal intensity profiles (blue color) of the laser pulses used for irradiating the sapphire sample; TL-pulse with a pulse duration of $30 \mathrm{fs}$ (left) and TOD-shaped pulse train (right), produced with a TOD phase mask, $\varphi^{(3)}=600 \times 10^{3} \mathrm{fs}^{3}$. (b) AFM micrographs overlaid with depth cross sections (black line) of two craters produced using a TL pulse at $89 \mathrm{~nJ}$ (left) and a TOD pulse train at $97 \mathrm{~nJ}$ (right). The arrows and dotted lines in $\mathrm{b}$ indicate the border of the different ablation regimes, evidenced as a slope change in the depth cross sections. (c) Squared diameter of the ablated zone as a function of the logarithm of the laser pulse energy for the different ablation regimes marked in $b$.

preserving the maximum depth of the generated structure. It is worth noting that the actual crater depth is likely even deeper, but the AFM tip is too wide to be able to probe this depth. The narrow crater shown produced by TOD shaped pulse in Figure $1 \mathrm{~b}$ features an inner crater radius $d=125 \mathrm{~nm}$, which predicts with a conservative estimation (Experimental Section) a maximum measurable depth of $d=270 \mathrm{~nm}$. The fact that actual value obtained from the measurement is higher is due to the conservative assumption made above, using the largest angle of the AFM tip for the estimation. The important point is that the AFM measurements made on such narrow structures might underestimate the actual depth, giving a lower bound of depth values. However, we have checked that this potential problem only affects a few nanopits in sapphire near the threshold fluence and does not compromise the message of our paper because it does not rely on absolute depth measurements but rather focuses on strategies to fabricate different shapes of narrow surface structures. We are currently working on implementing a method to provide correct depth values, by fabricating a replica as described in ref 34 .

The different ablation regimes induced by the TOD-shaped pulse shown in Figure $1 \mathrm{~b}$ can be identified as strong (red arrow) and gentle (blue arrows) ablation. Gentle ablation is a low-fluence-induced mechanism in which electron emission from the surface under ultrashort laser pulse excitation leads to the creation of an equal amount of positive charges at the target. Above a certain net charge density $\left(n_{\mathrm{ch}} \sim 10^{21} \mathrm{~cm}^{-3}\right)$ of positive ions at the surface, a strong electric field $\mathrm{E}$ is created, which is directed away from the target and can lead to breaking of atomic bonds for values of $\mathrm{E}>\mathrm{E}_{\mathrm{th}}\left(\mathrm{E}_{\mathrm{th}}=5 \times 10^{10} \mathrm{~V} / \mathrm{m}\right.$ in sapphire $\left.{ }^{35}\right)$. This eventually leads to ejection of positive ions in a fast, explosive process known as Coulomb explosion (CE). In a recent work using fs pulses with different pulse durations/ shapes and loose focusing, it has been shown that gentle ablation can be enhanced by pulse temporal shaping, yielding slightly larger crater depths. ${ }^{21}$ By studying the ablation dynamics in sapphire using fs-resolved microscopy, changes in the energy coupling behavior were observed and attributed to changes in the dominant ionization mechanisms caused by the temporal shape of the laser. For the TOD-shaped pulses used here, free carrier excitation and AI are enhanced with respect to MPI, leading to the generation of free carriers with higher kinetic energy (a seed and heat process, explained in refs 15 and 16 for fused silica and in ref 21 for sapphire) that favors $\mathrm{CE}^{21}$ The observed behavior is consistent with previous results $^{21}$ on the ablation dynamics of sapphire under fs-laser pulse irradiation.

The graphs in Figure 1c show the evolution of the square of the crater diameter as a function of the natural logarithm of the pulse energy. This is a standard method for determining the laser intensity distribution and diameter at the sample plane ${ }^{36}$ and also enables to infer, by extrapolation, the corresponding ablation thresholds. The linear fits included in the figure show a high correlation coefficient for both TL pulses and TODshaped pulses data sets. The extrapolated fluence threshold obtained for the TL pulse is $F_{\mathrm{TL}}=5.0 \mathrm{~J} / \mathrm{cm}^{2}$. The estimated thresholds for gentle and strong ablation (GA and SA, respectively) produced using TOD-shaped pulses (see fluences in Table 1) show a relative increase: $\Delta F / F_{\mathrm{TL}}$ of $83 \%\left(F_{\mathrm{TOD}, \mathrm{GA}}=\right.$

Table 1. Relation between the Different Temporal Pulse Shapes Used and the Corresponding Ablation Regime, Along with Their Characteristic Fluence Thresholds in Sapphire

\begin{tabular}{lllc}
\multicolumn{1}{c}{ pulse shape } & TOD $\left(\mathrm{fs}^{3}\right)$ & ablation regime & $\begin{array}{c}\text { fluence threshold } \\
\left(\mathrm{J} / \mathrm{cm}^{2}\right)\end{array}$ \\
transform-limited & 0 & strong ablation & 5.0 \\
pulse train & $600 \times 10^{3}$ & gentle ablation & 9.2 \\
pulse train & $600 \times 10^{3}$ & strong ablation & 10.1 \\
\hline
\end{tabular}

$\left.9.2 \mathrm{~J} / \mathrm{cm}^{2}\right)$ and $100 \%\left(F_{\mathrm{TOD}, S \mathrm{~A}}=10.1 \mathrm{~J} / \mathrm{cm}^{2}\right)$. We find that for both the TL pulse and the TOD-pulse train the same $1 / \mathrm{e}^{2}$ diameter value, $D_{\mathrm{TL}, \mathrm{GA}}=D_{\mathrm{TOD}, \mathrm{GA}}=2.0 \mu \mathrm{m}$, is obtained in the GA regime. This indicates that the nominal diffraction-limited diameter expected in the experiment $\left(D_{\text {nominal }}=1.4 \mu \mathrm{m}\right.$, Experimental Section) is not achieved either because $\mathrm{M}^{2}>1$ for the laser beam or a slight beam defocusing $\left(\mathrm{M}^{2}\right.$ is the beamquality factor; it denotes the degree of deviation the laser beam has with respect to an $\mathrm{M}^{2}=1$ ideal diffraction-limited Gaussian beam). Alternatively, for pulse-train-generated craters in the strong ablation regime, the fluence-dependence shows a considerably different slope (yet still Gaussian), yielding $D_{\text {TOD,SA }}=1.0 \mu \mathrm{m}$. The effective beam profile in the SA regime for the TOD-shaped pulse is thus different from that of a TL pulse, which is indicative of a change in the ablation mechanism. This further supports the idea that excitation mechanisms can be modified by changing the temporal 
distribution of the laser pulse and provides an explanation for the ability of such pulses for producing nanostructures below the diffraction limit. ${ }^{7,14-16,21,37}$ Although other approaches exist that allow fabrication of nanopits in dielectrics, they do have considerable drawbacks. Colloidal-particle lithography employing transform-limited fs laser pulses leads to nanopits with a diameter of down to $80 \mathrm{~nm}$ in soda-lime glass but also featuring a strong presence of debris, and it is inherently limited to the fabrication of hexagonal patterns. ${ }^{38}$ While, fabrication of nanopits in glasses using focused ion beam (FIB) techniques is doubtless superior in terms of achievable feature resolution, with $8 \mathrm{~nm}$ pores being reported recently in silica membranes, the complexity and slow throughput of FIB excludes it from the list of candidates for rapid precision manufacturing for industrial applications. ${ }^{39}$

Possible applications of nanopits fabricated with temporally shaped laser pulses could be the production of large-scale (arrays) low-cost molds for the fabrication of bioinspired, selfcleaning superhydrophobic polymeric surfaces (i.e., using soft lithography with urethane polymer ${ }^{40}$ ).

3.2. Phosphate Glass. Our choice of this phosphate glass for nanostructuring was motivated by recent works reporting surface swelling upon irradiation with loosely focused fs-laser pulses $(120 \mathrm{fs}$, spot diameter $D \approx 100 \mu \mathrm{m})$ at fluences below the ablation threshold. ${ }^{2,23}$ Surface swelling was attributed to an expansion of the molten/resolidified glass network, leading to a significant decrease of density (rarefaction) and refractive index $\left(\Delta n_{\mathrm{o}} / n_{\mathrm{o}}<-1 \%\right){ }^{22,23}$ On the basis of this material peculiarity, we have performed a study using tightly focused $30 \mathrm{fs}$ TL pulses and TOD-shaped pulses. Figure 2a shows the AFM map
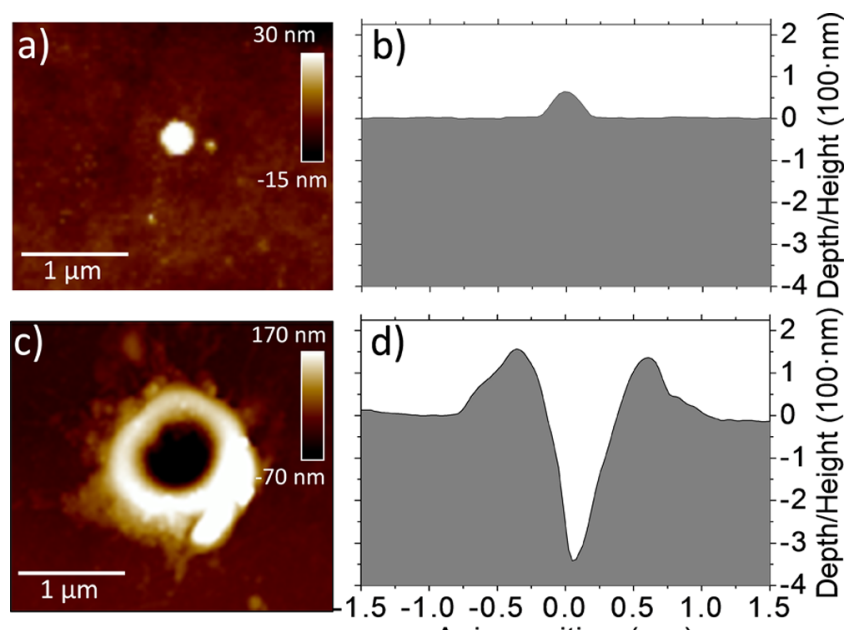

Axis position $(\mu \mathrm{m})$

Figure 2. ( $a$ and $c$ ) AFM micrographs and (b and d) depth cross sections for nanostructures produced in phosphate glass using a TL pulse at fluences below ( $\mathrm{a}$ and $\mathrm{b}, 30 \mathrm{~nJ}$ ) and above ( $\mathrm{c}$ and $\mathrm{d}, 49 \mathrm{~nJ})$ the ablation threshold of $39 \mathrm{~nJ}$.

of a region exposed to a single TL pulse below the ablation fluence threshold, featuring a nanobump. It can be seen in the corresponding topography profile along the center (Figure $2 \mathrm{~b}$ ) that the lateral extension $\left(D_{\text {bump(fwhm })}=242 \mathrm{~nm}\right)$ is well below the beam spot size and its height is considerable $\left(h_{\text {bump }}=+70\right.$ $\mathrm{nm})$. Other authors reported organized nanobump arrays (standard microscope slides) produced in glass by using fs-laser pulses combined with a polystyrene microsphere matrix. ${ }^{27}$ In that particular case, the feature size of the nanobumps $(h<100$ $\mathrm{nm}$, diameter $\approx 1 \mu \mathrm{m}$ ) were of the same order of magnitude as presented here. However, their fabrication required a complex previous step in the form of preparation of a colloidal monolayer, and their controllability strongly depends on the processing conditions and microsphere size plus matrix geometry. The approach presented here could be employed as a one-step simple method for the fabrication of nanolens arrays, whose features (diameter and curvature or height) can be tuned by tailoring the temporal intensity distribution of the laser.

Figure 2c shows an AFM map of a structure produced using a TL pulse at a fluence above the ablation threshold. For this fluence range, the irradiated area features a deep central crater surrounded by an elevated rim, resembling a nanovolcano. The corresponding topography profile (Figure $2 \mathrm{~d}$ ) reveals that the maximum crater depth is $h_{\text {volcano_crater }}=-360 \mathrm{~nm}$ and the rim reaches a height of $h_{\text {volcano rim }}=+145 \mathrm{~nm}$, yielding an overall depth of $\Delta h_{\text {volcano }}=-505 \mathrm{~nm}$. It is worth noting that for this structure the crater is less narrow than the one produced in sapphire; thus, the measured depth is most lightly not underestimated. The fact that the rim is higher than the nanobump shown in Figures 2 a,b suggests that bump-shaped nanostructures with larger heights can be fabricated by a slight increase of the laser fluence. We have confirmed this assumption experimentally by obtaining bumps with height values equal to those of the rims (bumps with $h_{\text {bump }} \approx 150 \mathrm{~nm}$ have been produced for TL pulses). However, we found that a height increase of nanobumps is unavoidably linked to an increase of their lateral size because of the underlying formation mechanism being resolidification of a laser-molten region into a less dense lattice structure. Its subablative nature also implies that the fabrication of nanobumps is constrained in a very narrow fluence window.

We have explored the influence of the TOD coefficient on the features of nanovolcano structures. Figure 3 shows several representative AFM maps of irradiated regions using different TOD coefficients $\left(0-700 \times 10^{3} \mathrm{fs}^{3}\right) \cdot{ }^{22,23}$ The irradiation fluences were chosen closely above the ablation threshold (for each temporal pulse shape) in order to compare different vertical features for craters with a similar lateral extension. The overall appearance in terms of the lateral extension of rim and crater is quite similar for all TOD values. The neatest structure with the lowest amount of debris surrounding the crater is observed for a TOD value of $\varphi^{(3)}=700 \times 10^{3} \mathrm{fs}^{3}$, suggesting that this pulse shape is optimal for fabricating sharp nanovolcanoes. As for the vertical dimension of the different topography maps, the considerable difference in image contrast already points out changes in the rim height. We have performed a detailed study, statistically analyzing the rim heights of 30 nanovolcanoes for each TOD coefficient. We have found that the rim height produced by a pulse with a given TOD coefficient is constant for pulse energies above the ablation threshold. This fact allowed us to determine a mean value for the rim height from an analysis of all nanovolcanoes (for a given TOD value) produced using different energies. The results are shown in Figure 4. The initially elevated rim height $(h=140 \pm 10 \mathrm{~nm})$ for TL pulses $\left(0 \mathrm{fs}^{3}\right)$ features a sharp decrease to $h \approx 75 \mathrm{~nm}$ by adding a relatively small amount of TOD $\left(100-300 \times 10^{3} \mathrm{fs}^{3}\right)$. A further increase to $600 \times 10^{3} \mathrm{fs}^{3}$ and up to $1000 \times 10^{3} \mathrm{fs}^{3}$ leads to a height increase well beyond the values obtained by TL pulses.

To enable a quantitative comparison between the results obtained using TOD-shaped pulse trains and single TL laser 

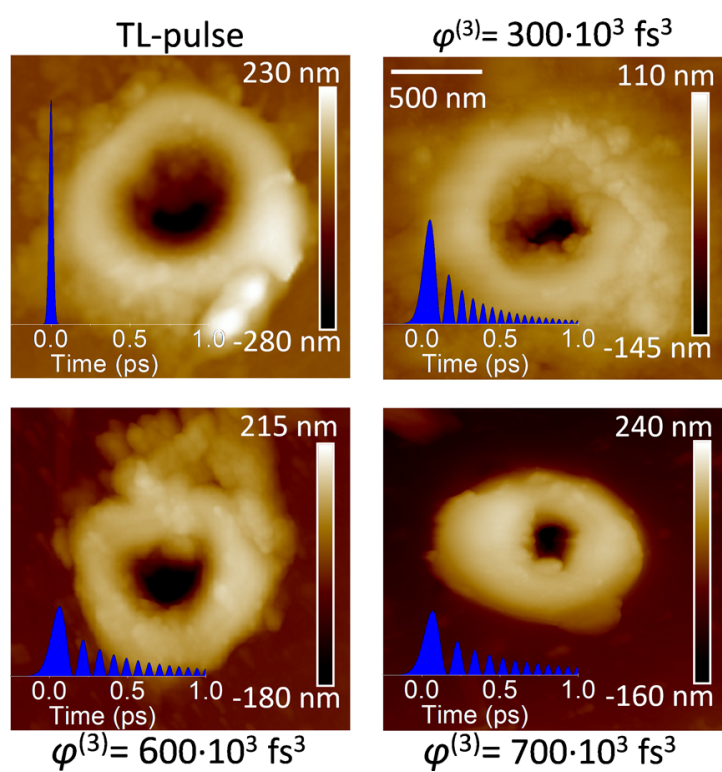

Figure 3. AFM maps of nanovolcanoes produced in phosphate glass using temporally shaped fs-laser pulses with increasing TOD coefficients; clockwise from top left, $\varphi^{(3)}=0 \mathrm{fs}^{3}$ (for a TL pulse, 49 $\mathrm{nJ}$ pulse energy), $300 \times 10^{3} \mathrm{fs}^{3}(86 \mathrm{~nJ}), 600 \times 10^{3} \mathrm{fs}^{3}(121 \mathrm{~nJ})$, and $700 \times 10^{3} \mathrm{fs}^{3}(122 \mathrm{~nJ})$. The insets show the temporal intensity profiles of the laser pulses. The structures shown are illustrative of the observed features for the TOD values and were chosen in terms of sharpness rather than threshold.

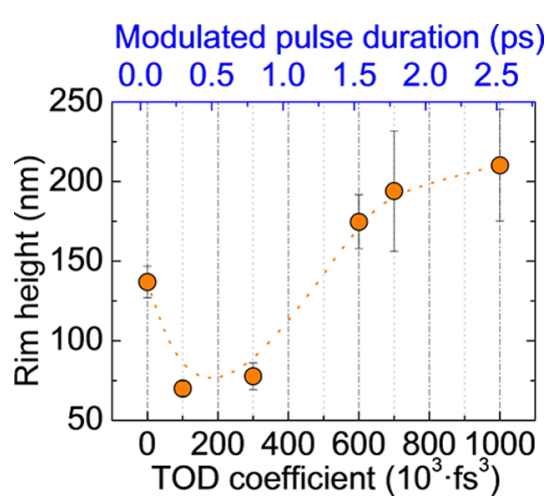

Figure 4. Height of the crater rim as a function of the TOD coefficient. The values were averaged for 30 irradiated regions above the ablation threshold with saturated rim height. The upper $x$ axis shows the modulated pulse duration, corresponding to the duration of the pulse intensity envelope. ${ }^{16}$

pulses, we have calculated the modulated pulse duration $\Delta t_{\text {mod }}$ which can be understood as the duration of the pulse intensity envelope of the pulse train. To this end, we have made use of the simplified formula: $\Delta t_{\text {mod }} \approx 2.31\left(\varphi^{(3)} / \Delta t^{2}\right)$, where $\varphi^{(3)}$ is the TOD coefficient and $\Delta t$ is the pulse duration (fwhm) for a TL pulse (this approximation is valid because $\varphi^{(3)}>\Delta t^{3}$ for every TOD value employed). ${ }^{16}$ The extracted values have been included in Figure 4 as an upper axis and show that the modulated pulse duration of TOD-shaped pulses is quite long even for low TOD coefficients. Although a definitive explanation for the observed increase of the rim height with TOD coefficients above $100 \times 10^{3} \mathrm{fs}^{3}$ cannot be given with the available data, we can provide a hypothesis for this behavior. The different temporal pulse shapes, associated with the different TOD values employed, lead to a different excitation dynamics. As a consequence, free electrons produced by the leading part of the laser pulse train via MPI ${ }^{13,16,41}$ experience significant and long-lasting heating and acceleration by the long tail of the pulse, leading to impact ionization over a prolonged time. In particular, pulses produced with high TOD values favor electron acceleration and impact ionization. We speculate that the resulting free electrons with high kinetic energy penetrate deeper into the material and thus lead to larger melt depth. As a consequence, upon resolidification into a less dense structure, the absolute volume increase is larger, leading to higher rims.

Similar hollow nanostructures, nanobowls, and nanovolcanoes have been generated using other fabrication methods in different materials. Large-sized ordered surface arrays of bowllike $\mathrm{TiO}_{2}$ units were obtained using colloidal-particle lithography combined with ion-beam milling, atomic layer deposition, and etching. ${ }^{24}$ Hollow nanovolcanoes were fabricated in photoresist with high controllability by exploiting the optical near and far field of a colloidal monolayer on a thick photoresist layer to etch 3D structures. ${ }^{25}$ Yet, a strong point of our approach compared to the above-mentioned techniques is its ability to produce these nanostructures in a single-step process. Such structures can be used for nanoparticle trapping, as seen in ref 25 , and could be further functionalized for applications in drug delivery and biotrapping (already investigated in hollow silica nanoparticles). ${ }^{42}$

\section{CONCLUSIONS}

In this manuscript, the use of ultrashort pulse trains generated by third-order dispersion has been demonstrated to be a powerful tool for surface nanofabrication in dielectrics. This approach, combined with a smart material selection according to the specific response to ultrashort laser pulse irradiation, enables the fabrication of a variety of technologically relevant nanostructures, including nanopits (sapphire), nanobumps (phosphate glass) and nanovolcanoes (phosphate glass).

We have found that for sapphire, clean nanoscale processing is possible without melt-flow or splashing effects. It has been shown that it is feasible to reduce the diameter of the soproduced nanopits by a factor of four while preserving the crater depth when using TOD-shaped pulses instead of TL pulses. The use of TOD-shaped pulses allows control of the material ejection mechanisms in sapphire, ranging from gentle to strong ablation. For phosphate glass, nanobumps can be produced at subablation fluences. Above the ablation threshold, the irradiated region shows a volcanolike shape, and the height of the rim surrounding the central ablated hole can be controlled by tuning the TOD coefficient.

\section{AUTHOR INFORMATION}

\section{Corresponding Authors}

*E-mail: fjavihr@gmail.com.

*E-mail: j.siegel@io.cfmac.csic.es.

\section{Notes}

The authors declare no competing financial interest.

\section{ACKNOWLEDGMENTS}

This work has been partially supported by the Spanish MINECO projects TEC2011-22422 and MAT2013-33517. J.H.-R. acknowledges the FPI grant and the FPI-EEBB travel grant awarded by the Spanish Ministry of Science and Innovation. N.G., B.Z., C.S., M.W., and T.B. acknowledge support from the DFG priority program no. 1327. 


\section{REFERENCES}

(1) Davis, K.; Miura, K.; Sugimoto, N.; Hirao, K. Writing Waveguides in Glass with a Femtosecond Laser. Opt. Lett. 1996, 21, 1729-1731.

(2) Sugioka, K., Cheng, Y., Eds. Ultrafast Laser Processing: Micro to Nanoscale; Pan Stanford Publishing: Singapore, 2013.

(3) Osellame, R.; Cerullo, G.; Ramponi, R. Femtosecond Laser Micromachining: Photonic and Microfluidic Devices in Transparent Materials; Top. Appl. Phys. Series; Asheron, C., Dresselhaus, M. S., Eds.; Springer-Verlag: Berlin Heidelberg, Germany, 2012; Vol. 123

(4) Rajput, D.; Costa, L.; Lansford, K.; Terekhov, A.; Hofmeister, W. Solution-Cast High-Aspect-Ratio Polymer Structures from DirectWrite Templates. ACS Appl. Mater. Interfaces 2013, 5, 1-5.

(5) Yong, J.; Chen, F.; Yang, Q.; Du, G.; Bian, H.; Zhang, D.; Si, J.; Yun, F.; Hou, X. Rapid Fabrication of Large-Area Concave Microlens Arrays on PDMS by a Femtosecond Laser. ACS Appl. Mater. Interfaces 2013, 5, 9382-9385.

(6) Joglekar, A. P.; Liu, H.-H.; Meyhöfer, E.; Mourou, G.; Hunt, A. J. Optics at critical Intensity: Applications to Nanomorphing. Proc. Natl. Acad. Sci. U.S.A. 2004, 101, 5856-5861.

(7) Englert, L.; Wollenhaupt, M.; Sarpe, C.; Otto, D.; Baumert, T. Morphology of Nanoscale Structures on Fused Silica Surfaces from Interaction with Temporally Tailored Femtosecond Pulses. J. Laser Appl. 2012, 24, 042002.

(8) (a) Bachau, H.; Belsky, A. N.; Bogatyrev, I. B.; Gaudin, J.; Geoffroy, G.; Guizard, S.; Martin, P.; Popov, Y. V.; Vasil'ev, A. N.; Yatsenko, B. N. Electron Heating Through a Set of Random Levels in the Conduction Band of Insulators Induced by Femtosecond Laser Pulses. Appl. Phys. A: Mater. Sci. Process. 2010, 98, 679-689. (b) Mao, S. S.; Quéré, F.; Guizard, S.; Mao, X.; Russo, R. E.; Petite, G.; Martin, P. Dynamics of Femtosecond Laser Interactions with Dielectrics. Appl. Phys. A: Mater. Sci. Process. 2004, 79, 1695-1709.

(9) Puerto, D.; Siegel, J.; Gawelda, W.; Galvan-Sosa, M.; Ehrentraut, L.; Bonse, J.; Solis, J. Dynamics of Plasma Formation, Relaxation, and Topography Modification Induced by Femtosecond Laser Pulses in Crystalline and Amorphous Dielectrics. J. Opt. Soc. Am. B 2010, 27, $1065-1076$.

(10) Hernandez-Rueda, J.; Puerto, D.; Siegel, J.; Galvan-Sosa, M.; Solis, J. Plasma Dynamics and Structural Modifications Induced by Femtosecond Laser Pulses in Quartz. Appl. Surf. Sci. 2012, 258, 93899393.

(11) Sarpe, C.; Köhler, J.; Winkler, T.; Wollenhaupt, M.; Baumert, T. Real Time Observation of Transient Electron Density in Water Irradiated with Tailored Femtosecond Laser Pulses. New J. Phys. 2012, 14, 075021 .

(12) Sarpe-Tudoran, C.; Assion, A.; Wollenhaupt, M.; Winter, M.; Baumert, T. Plasma Dynamics of Water Breakdown at a Water Surface Induced by Femtosecond Laser Pulses. Appl. Phys. Lett. 2006, 88, 26110.

(13) Hernandez-Rueda, J.; Siegel, J.; Puerto, D.; Galvan-Sosa, M.; Gawelda, W.; Solis, J. Ad-hoc Design of Temporally Shaped Fs Laser Pulses Based on Plasma Dynamics for Deep Ablation in Fused Silica. Appl. Phys. A: Mater. Sci. Process. 2012, 112, 185-189.

(14) Englert, L.; Rethfeld, B.; Haag, L.; Wollenhaupt, M.; SarpeTudoran, C.; Baumert, T. Control of Ionization Processes in High Band Gap Materials via Tailored Femtosecond Pulses. Opt. Express 2007, 15, 17855-17862.

(15) Englert, L.; Wollenhaupt, M.; Haag, L.; Sarpe-Tudoran, C.; Rethfeld, B.; Baumert, T. Material Processing of Dielectrics with Temporally Asymmetric Shaped Femtosecond Laser Pulses on the Nanometer Scale. Appl. Phys. A: Mater. Sci. Process. 2008, 92, 749-753.

(16) Wollenhaupt, M.; Englert, L.; Horn, A.; Baumert, T. Temporal Femtosecond Pulse Tailoring for Nanoscale Laser Processing of WideBandgap Materials. Proc. SPIE 2010, 7600, 1-11.

(17) Mermillod-Blondin, A.; Mauclair, C.; Rosenfeld, A.; Bonse, J.; Hertel, I.; Audouard, E.; Stoian, R. Size Correction in Ultrafast Laser Processing of Fused Silica by Temporal Pulse Shaping. Appl. Phys. Lett. 2008, 93, 021921-021924.
(18) Stoian, R.; Boyle, M.; Thoss, A.; Rosenfeld, A.; Korn, G.; Hertel, I. V. Laser Ablation of Dielectrics with Temporally Shaped Femtosecond Pulses. Appl. Phys. Lett. 2002, 80, 353-355.

(19) Rebollar, E.; Mildner, J.; Götte, N.; Otto, D.; Sarpe, C.; Köhler, J.; Wollenhaupt, M.; Baumert, T.; Castillejo, M. Microstructuring of Soft Organic Matter by Temporally Shaped Femtosecond Laser Pulses. Appl. Surf. Sci. 2014, 302, 231-235.

(20) Ben-Yakar, A.; Harkin, A.; Ashmore, J.; Byer, R. L.; Stone, H. Thermal and Fluid Processes of a Thin Melt Zone during Femtosecond Laser Ablation of Glass: the Formation of Rims by Single Laser Pulses. J. Phys. D: Appl. Phys. 2007, 40, 1447-1459.

(21) Hernandez-Rueda, J.; Siegel, J.; Galvan-Sosa, M.; Ruiz de la Cruz, A.; Garcia-Lechuga, M.; Solis, J. Controlling Ablation Mechanisms in Sapphire by Tuning the Temporal Shape of Fs-Laser Pulses. J. Opt. Soc. Am. B 2015, 32, 150-156.

(22) Puerto, D.; Siegel, J.; Ferrer, A.; Hernandez-Rueda, J.; Solis, J. Correlation of the Refractive Index Change at the Surface and Inside Phosphate Glass upon Femtosecond Laser Irradiation. J. Opt. Soc. Am. B 2012, 29, 2665-2668.

(23) Hernandez-Rueda, J.; Siegel, J.; Garcia-Lechuga, M.; Solis, J. Femtosecond Laser-Induced Refractive Index Changes at the Surface of Dielectrics: Quantification Based on Newton Ring Analysis. J. Opt. Soc. Am. B 2014, 31, 1676-1683.

(24) Wang, X. D.; Graugnard, E.; King, J. S.; Wang, Z.; Summers, C. J. Large-Scale Fabrication of Ordered Nanobowl Arrays. Nano Lett. 2004, 4, 2223-22261.

(25) Zhang, X. A.; Elek, J.; Chang, C.-H. Three-Dimensional Nanolithography Using Light Scattering from Colloidal Particles. ACS Nano 2013, 7, 6212-6218.

(26) Meng, X.; Zhang, X.; Ye, L.; Qiu, D. Fabrication of Large-Sized Two-Dimensional Ordered Surface Array with Well-Controlled Structure via Colloidal Particle Lithography. Langmuir 2014, 30, 7024-7029.

(27) Bityurin, N.; Afanasiev, A.; Bredikhin, V.; Alexandrov, A.; Agareva, N.; Pikulin, A.; Ilyakov, I.; Shishkin, B.; Akhmedzhanov, R. Colloidal Particle Lens Arrays-Assisted Nanopatterning by Harmonics of a Femtosecond Laser. Opt. Express 2013, 21, 21485.

(28) Ashkenasi, D.; Lorenz, M.; Stoian, R.; Rosenfeld, A. Surface damage threshold and structuring of dielectrics using femtosecond laser pulses: the role of incubation. Appl. Surf. Sci. 1999, 150, 101-106.

(29) Krüger, J.; Kautek, W. Ultrashort Pulse Laser Interaction with Dielectrics and Polymers. Adv. Polym. Sci. 2004, 168, 247-289.

(30) (a) Köhler, J.; Wollenhaupt, M.; Bayer, T.; Sarpe, C.; Baumert, T. Zeptosecond Precision Pulse Shaping. Opt. Express 2011, 19, 11638-11653. (b) Wollenhaupt, M.; Krug, M.; Köhler, J.; Bayer, T.; Sarpe-Tudoran, C.; Baumert, T. Photoelectron Angular Distributions From Strong-Field Coherent Electronic Excitation. Appl. Phys. B: Lasers Opt. 2009, 95, 245-259.

(31) Weiner, A. M. Femtosecond Pulse Shaping Using Spatial Light Modulators. Rev. Sci. Instrum. 2000, 71, 1929-1960.

(32) Richards, B.; Wolf, E. Electromagnetic Diffraction in Optical Systems. II. Structure of the Image Field in an Aplanatic Systems. Proc. R. Soc. London, Ser. A 1959, 253, 358-379.

(33) Urbain, G.; Bottinga, Y.; Richet, P. Viscosity of Liquid Silica, Silicates and Alumino-Silicates. Geochim. Cosmochim. Acta 1982, 46, 1061-1072.

(34) White, Y. W.; Li, X.; Sikorski, Z.; Davis, L. M.; Hofmeister, W. Single-Pulse Ultrafast-Laser Machining of High Aspect Nano-Holes at the Surface of $\mathrm{SiO}_{2}$. Opt. Express 2008, 16, 14411-14420.

(35) Bulgakova, N. M.; Stoian, R.; Rosenfeld, A.; Hertel, I. V.; Campbell, E. E. B. Electronic Transport and Consequences for Material Removal in Ultrafast Laser Ablation of Materials. Phys. Rev. B: Condens. Matter Mater. Phys. 2004, 69, 054102.

(36) Liu, J. M. Simple Technique for Measurements of Pulsed Gaussian-Beam Spot Sizes. Opt. Lett. 1982, 7, 196-199.

(37) Ashkenasi, D.; Rosenfeld, A.; Varel, H.; Wähmer, M.; Campbell, E. E. B. Laser Processing of Sapphire with Picosecond and SubPicosecond Pulses. Appl. Surf. Sci. 1997, 120, 65-80. 
(38) Sakai, T.; Nedyalkov, N.; Obara, M. Positive and Negative Nanohole-Fabrication on Glass Surface by Femtosecond Laser with Template of Polystyrene Particle Array. J. Phys. D: Appl. Phys. 2007, 40, 2102-2107.

(39) Hemamouche, A.; Morin, A.; Bourhis, E.; Toury, B.; Tarnaud, E.; Mathé, J.; Guégan, P.; Madouri, A.; Lafosse, X.; Ulysse, C.; Guilet, S.; Patriarche, G.; Auvray, L.; Montel, F.; Wilmart, Q.; Plaçais, B.; Yates, J.; Gierak, J. FIB Patterning of Dielectric, Metallized and Graphene Membranes: A Comparative Study. Microelectron. Eng. 2014, 121, 87-91.

(40) Bixler, G. D.; Bhushan, B. Rice- and Butterfly-Wing Effect Inspired Self-Cleaning and Low Drag Micro/Nanopatterned Surfaces in Water, Oil, and Air Flow. Nanoscale 2014, 6, 76-96.

(41) Rethfeld, B. Free-Electron Generation in Laser-Irradiated Dielectrics. Phys. Rev. B: Condens. Matter Mater. Phys. 2006, 73, 035101.

(42) Li, Z.-Z.; Wen, L.-X.; Shao, L.; Chen, J.-F. Fabrication of Porous Hollow Silica Nanoparticles and their Applications in Drug Release Control. J. Controlled Release 2004, 98, 245-254. 